\title{
CLORETO DE MEPIQUAT, THIDIAZURON E ETHEPHON APLICADOS NO ALGODOEIRO EM PONTA PORÃ, MS ${ }^{1}$
}

\author{
FERNANDO MENDES LAMAS ${ }^{2}$, MANOEL LUIZ FERREIRA ATHAYDE ${ }^{3}$ \\ DAVID ARIOVALDO BANZATTO ${ }^{4}$ e PAULO DE ANDRADE FORTUNA ${ }^{5}$
}

\begin{abstract}
RESUMO - O objetivo do presente estudo foi avaliar os efeitos de diferentes dosagens de cloreto de mepiquat, thidiazuron e ethephon, aplicadas parceladamente no algodoeiro (Gossypium hirsutum L.) na Fazenda Itamarati, Ponta Porã, MS. As dosagens de cloreto de mepiquat foram: $(0 ; 12,5+12,5+25,0=50 ; 25+25+25=75 ; 0+50+50=100 ; 12,5+62,5+50=125) \mathrm{g} \mathrm{ha}^{-1}, \mathrm{com}$ aplicações efetuadas aos 34, 47 e 62 dias após a emergência (DAE) em 1993/94, e aos 42, 60 e 73 DAE, em 1994/95, enquanto o thidiazuron foi aplicado quando $70 \%$ dos capulhos estavam abertos, nas dosagens de $0,45,60$ e $75 \mathrm{~g} \mathrm{ha}^{-1}$; já o ethephon foi aplicado sete dias após o thidiazuron, quando já se observava desfolha de $85 \%$, nas dosagens de 0,960 e $1.440 \mathrm{~g} \mathrm{ha}^{-1}$. O delineamento experimental utilizado foi o de blocos casualizados em faixa, com subparcelas subdivididas e quatro repetições. $\mathrm{O}$ cloreto de mepiquat proporcionou redução do número de frutos verdes, aumento do peso de 100 sementes e do peso médio de um capulho; a percentagem de desfolha aumentou com as dosagens de thidiazuron e ethephon; constatou-se que a interação cloreto de mepiquat $\mathrm{x}$ thidiazuron $\mathrm{x}$ ethephon foi significativa para percentagem de abertura de capulhos e produção de algodão em caroço.
\end{abstract}

Termos para indexação: Gossypium hirsutum, regulador de crescimento, desfolhante, maturador.

\author{
MEPIQUAT CHLORIDE, THIDIAZURON AND ETHEPHON APPLIED
}

ON COTTON IN PONTA PORÃ, MS, BRAZIL

\begin{abstract}
The objective of this study was to evaluate the effect of doses of mepiquat chloride, thidiazuron and ethephon on cotton (Gossypium hirsutum L.), applied in parcels, and were surveyed in Itamarati Farm at Ponta Porã county. The mepiquat chloride doses were: $(0.0 ; 12.5+$ $12.5+25.0=50.0 ; 25.0+25.0+25.0=75.0 ; 0.0+50.0+50.0=100.0 ; 12.5+62.5+50.0=125.0) \mathrm{g} \mathrm{ha}^{-1}$. The applications were made at 34, 47 and 63 days after emergence(DAE) in 1993/94 and at 42, 60 and 73 DAE in 1994/95. Thidiazuron was applied when $70 \%$ of bolls were opened at the doses $0.0,45.0$, 60.0 and $75.0 \mathrm{~g} \mathrm{ha}^{-1}$. Ethephon was applied seven days after thidiazuron, when $85 \%$ defoliation was observed, in the doses of $0.0,960.0$ and 1,440.0 $\mathrm{g} \mathrm{ha}^{-1}$. The experimental design used was strips with subdivided subparcelas. Mepiquat chloride decrease the number of unripe fruits, increased the weight of 100 seeds and the boll average weight. The defoliation percentage enhanced with increasing thidiazuron and ethephon doses. Mepiquat chloride $\mathrm{x}$ thidiazuron $\mathrm{x}$ ethephon interaction was significative to the percentage of opening bolls and production of cotton seed
\end{abstract}

Index terms: Gossypium hirsutum, growth regulator, defoliant, riper.

${ }^{1}$ Aceito para publicação em 9 de fevereiro de 1999.

Extraído da tese do primeiro autor, apresentada à Faculdade de Ciências Agrárias e Veterinárias da UNESP, Campus de Jaboticabal.

${ }^{2}$ Eng. Agr., Dr., Embrapa-Centro de Pesquisa Agropecuária do Oeste (CPAO), Caixa Postal, 661, CEP 79804-970 Dourados, MS. E-mail: lamas@embrapa.com.br
${ }^{3}$ Eng. Agr., Dr., Prof. Titular, Dep. de Fitotecnica, FCAVJ, UNESP, Rodovia Carlos Tonani, Km 5, CEP 14870-000 Jaboticabal, SP.

${ }^{4}$ Eng. Agr., Dr., Prof. Adjunto, Dep. de Ciências Exatas, FCAVJ, UNESP

${ }^{5}$ Eng. Agr., Aluno do curso de pós-graduação, FCAVJ, UNESP. 


\section{INTRODUÇÃO}

A utilização de produtos químicos que proporcionam alterações na arquitetura das plantas vem sendo incrementada na cultura do algodoeiro, especialmente quando se adota alto nível tecnológico. Entre eles, tem-se o cloreto de mepiquat (cloreto 1,1-dimetil piperídineo), utilizado visando a melhores condições de manejo, com redução da altura das plantas, e maior uniformização, facilitando a colheita mecanizada (Carvalho et al., 1994). Resultados de pesquisas com esse produto são relatados nos trabalhos de York (1983), Cia et al. (1984), Stuart et al. (1984), Reddy et al. (1990), Hodges et al. (1991), Reddy et al. (1992), Wallace et al. (1993) e Carvalho et al. (1994).

Produtos com efeito desfolhante e que antecipam a maturação dos frutos também vêm tendo uso crescente, destacando-se o thidiazuron (N - phenil - N'- 1,2,3 - thiadiazol - 5 - uréia) que, além de provocar a desfolha do algodoeiro, facilitando a colheita, especialmente a mecanizada, reduz a frutificação tardia e contribui para a redução do número de pragas que entram em diapausa e infestam lavouras no ano seguinte (Hopkins \& Moore, 1980; Bariola et al., 1990).

Segundo Suttle (1985), com a aplicação de thidiazuron verifica-se redução no nível e no transporte endógeno do ácido indol acético, inibidor da abscisão, resultando em substancial aumento da produção de etileno, hormônio responsável pela formação da camada de abscisão; a aplicação de auxina sintética (ácido naftaleno acético) inibe o efeito do etileno na abscisão de folhas (Suttle $\&$ Hultstrand, 1990).

A época de aplicação do thidiazuron pode influenciar significativamente os resultados. De acordo com trabalhos desenvolvidos por Thakral (1991) e Snipes \& Baskin (1994), que fizeram aplicações quando se tinha $20,40,60$ e $80 \%$ de capulhos abertos, em aplicações precoces, ou seja, com menos de $60 \%$ de capulhos abertos, houve redução significativa da produção de algodão em caroço e alterações negativas nas características tecnológicas da fibra.

Entre os fatores climáticos, a temperatura, conforme Kerby et al. (1988), é a que mais influencia os resultados da aplicação do produto e segundo eles, para que a eficiência do produto seja boa, é necessário, nos dias seguintes ao da sua aplicação, temperatura média superior a $20^{\circ} \mathrm{C}$. Resultados semelhantes também foram constatados por Bariola et al. (1990), que avaliaram o efeito do produto em diferentes temperaturas.

Trabalhos desenvolvidos em diferentes condições ecológicas revelaram que os melhores resultados foram obtidos com dosagens maiores (125 a $200 \mathrm{~g} \mathrm{ha}^{-1}$ ) dependendo, fundamentalmente, da temperatura (Malik et al., 1991; Thakral, 1991).

Visando reduzir o ciclo do algodoeiro, muitas vezes são utilizados produtos químicos com a finalidade de acelerar a maturação dos frutos, sua deiscência e a desfolha das plantas, aumentando a precocidade e contribuindo para diminuir os sítios de oviposição de lagarta-rosada, Pectinophora gossypiella (Saunders, 1844) e do bicudo, Anthonomus grandis (Boheman, 1843); entre esses produtos estão os chamados maturadores, utilizados na terminação química (Bariola \& Henneberry, 1986; Henneberry et al., 1988 e Montandon et al., 1994); quanto aos maturadores utilizados na cultura do algodoeiro, tem-se o ethephon (ácido 2 cloro etil fosfônico) substância liberadora de etileno; $\mathrm{o}$ etileno inibe a biossíntese e, conseqüentemente, a movimentação de auxinas (Warner \& Leopold, 1969); a aplicação do ethephon proporciona a redução do número de maçãs verdes, de botões florais e flores (Bariola \& Henneberry, 1986; Montandon et al., 1994).

Pettigrew et al. (1992) fizeram aplicações de ethephon em plantas com aproximadamente 56 dias de emergência, observando acentuada abscisão de estruturas frutíferas, redução no tamanho dos capulhos, efeito depressivo sobre o índice micronaire, maturidade de fibras e sobre a produção de fibras.

Em trabalhos desenvolvidos por Jones et al. (1990), foram avaliadas as dosagens de $0,0,0,14,0,28$, 0,56 e $0,84 \mathrm{~kg} \mathrm{ha}^{-1}$ de ethephon, aplicadas na fase final de floração e se concluiu que as dosagens de 0,56 e $0,84 \mathrm{~kg} \mathrm{ha}^{-1}$ causaram significativa abscisão de frutos três dias após a aplicação; no entanto, a produção de algodão em caroço não foi afetada. $\mathrm{O}$ efeito do produto sobre a produção de algodão em 
caroço e sobre as características tecnológicas da fibra é altamente dependente da época de aplicação (Smith et al., 1988).

Vários são os trabalhos desenvolvidos com os produtos cloreto de mepiquat, thidiazuron e ethephon aplicados de forma isolada; entretanto, a interação entre esses produtos não tem sido objeto de estudo.

O objetivo do presente trabalho foi estudar os efeitos de diferentes dosagens de cloreto de mepiquat, aplicadas de maneira parcelada, de thidiazuron e de ethephon e a interação entre eles sobre algumas características do algodoeiro.

\section{MATERIAL E MÉTODOS}

Os experimentos foram conduzidos na Fazenda Itamarati, localizada em Ponta Porã, MS, com altitude média de $630 \mathrm{~m}$, e coordenadas geográficas de $22^{\circ} 30^{\prime} \mathrm{de}$ latitude $\mathrm{S}$ e $54^{\circ} 44^{\prime}$ de longitude $\mathrm{O}$, durante os anos agrícolas de 1993/94 e 1994/95. Utilizou-se a cultivar CNPA ITA 90 semeada no espaçamento de $0,76 \mathrm{~m}$ entre fileiras, com densidade de 15 sementes metro ${ }^{-1}$, resultando uma população de aproximadamente 130.000 plantas ha- $^{-1}$, no primeiro ano, a emergência foi em $5 / 11 \mathrm{e}$, no segundo, em $9 / 11$

Por ocasião da semeadura, foi realizada a seguinte adubação: $261 \mathrm{~kg} \mathrm{ha}^{-1}$ de MAP (fosfato monoamônico), $80 \mathrm{~kg} \mathrm{ha}^{-1}$ de superfosfato triplo, $50 \mathrm{~kg} \mathrm{ha}^{-1}$ de superfosfato simples, $150 \mathrm{~kg} \mathrm{ha}^{-1}$ de cloreto de potássio, $20 \mathrm{~kg} \mathrm{ha}^{-1} \mathrm{de}$ óxido de zinco, $14,3 \mathrm{~kg} \mathrm{ha}^{-1}$ de óxido de cobre e $10 \mathrm{~kg} \mathrm{ha}^{-1}$ de ulexita ( $12 \%$ de boro). A adubação de cobertura com $\mathrm{N}$ totalizou $149 \mathrm{~kg} \mathrm{ha}^{-1}$, aplicados parceladamente, via pivô central: $30+46+46+27 \mathrm{~kg} \mathrm{ha}^{-1} \mathrm{de} \mathrm{N}$, respectivamente aos 25, 50, 75 e 100 dias após a emergência (DAE)

O controle de plantas daninhas foi feito com a utilização de $1,5 \mathrm{~kg} \mathrm{ha}^{-1}$ de diuron, aplicado em pré-emergência, mais dois cultivos mecânicos e duas capinas manuais, de forma a se evitar a interferência das plantas daninhas com a cultura. Foram realizadas irrigações suplementares, com pivô central, sempre que necessário. O controle de pragas foi realizado com base em resultados de amostragens semanais, segundo Santos (1993).

O delineamento experimental utilizado foi o de blocos casualizados, em faixa, com subparcelas subdivididas e quatro repetições (Farias, 1984). Em 1993/94, nas parcelas foram alocadas as dosagens de cloreto de mepiquat (CM), nas subparcelas as dosagens de ethephon (ETH) e nas subsubparcelas as dosagens de thidiazuron (TDZ). As dosagens de cloreto de mepiquat e o esquema de parcelamento adotado encontram-se na Tabela 1; as dosagens de ethephon estudadas foram: 0,960 e $1.440 \mathrm{~g} \mathrm{ha}^{-1}$ e as de thidiazuron: $0,45,60 \mathrm{e} 75 \mathrm{~g} \mathrm{ha}^{-1}$. As faixas para aplicação do cloreto de mepiquat constaram da área de $27.360 \mathrm{~m}^{2}(45,6 \times 600 \mathrm{~m})$, e nas subparcelas em que se aplicou o ethephon a área foi de $9.120 \mathrm{~m}^{2}(15,2 \times 600 \mathrm{~m})$ enquanto a das subsubparcelas que receberam os tratamentos com thidiazuron foi de $231,04 \mathrm{~m}^{2}(15,2 \times 15,2 \mathrm{~m})$. Em 1994/95, o ethephon não foi aplicado, portanto, utilizou-se o delineamento experimental de blocos casualizados em faixa, e quatro repetições; as parcelas que receberam as dosagens de cloreto de mepiquat, $27.360 \mathrm{~m}^{2}(45,6 \times 600 \mathrm{~m})$ e as que receberam o thidiazuron, $231,04 \mathrm{~m}^{2}(15,2 \mathrm{x}$ $15,2 \mathrm{~m}$ ); como área útil foram consideradas as três fileiras centrais de cada subsubparcela $11,4 \mathrm{~m}^{2}(2,28 \times 5 \mathrm{~m})$ nos dois anos.

As aplicações de cloreto de mepiquat foram realizadas: 1) ano de 1993/94: a) primeira aplicação, 34 DAE, plantas com $51 \mathrm{~cm}$ de altura; b) segunda aplicação, 47 DAE, plantas com $84 \mathrm{~cm}$; c) terceira aplicação, 62 DAE, plantas com $93 \mathrm{~cm}$; 2) ano de 1994/95: a) primeira aplicação, 42 DAE, plantas com $61 \mathrm{~cm}$; b) segunda aplicação, 60 DAE, plantas com $79 \mathrm{~cm}$; c) terceira aplicação, 73 DAE, plantas com $97 \mathrm{~cm}$.

O thidiazuron foi aplicado quando aproximadamente $70 \%$ dos frutos estavam abertos, e o ethephon, sete dias após o thidiazuron, quando se verificou desfolha média de $85 \%$. Todos os produtos foram aplicados nas primeiras horas da manhã, com avião agrícola Ipanema, acoplado com barra de pulverização e vazão de 30 litros de calda por hectare

A colheita do experimento, nos dois anos, foi realizada manualmente, em uma única vez, com aproximadamente $90 \%$ de capulhos abertos

Dentro da área útil de cada unidade experimental, em três metros de fileira, imediatamente antes da colheita, foram avaliados: percentagem de desfolha, número de maçãs e percentagem de abertura de capulhos. A

TABELA 1. Tratamentos estudados e esquema de parcelamento das dosagens de cloreto de mepiquat.

\begin{tabular}{crrrr}
\hline \multirow{2}{*}{ Tratamentos } & \multicolumn{3}{c}{ Aplicações parceladas $\left(\mathrm{g} \mathrm{ha}^{-1} \mathrm{de}\right.$ i.a. $)$} & \multirow{2}{*}{ Total } \\
\cline { 2 - 3 } & \multicolumn{1}{c}{$1^{\mathrm{a}}$} & \multicolumn{1}{c}{$2^{\mathrm{a}}$} & $3^{\mathrm{a}}$ & \\
\hline 1 & 0,0 & 0,0 & 0,0 & 0,0 \\
2 & 12,5 & 12,5 & 25,0 & 50,0 \\
3 & 25,0 & 25,0 & 25,0 & 75,0 \\
4 & 0,0 & 50,0 & 50,0 & 100,0 \\
5 & 12,5 & 62,5 & 50,0 & 125,0 \\
\hline
\end{tabular}

Pesq. agropec. bras., Brasília, v.34, n.10, p.1871-1880, out. 1999 
produção de algodão em caroço foi obtida pelo peso do algodão em caroço colhido das plantas dentro da área útil, cujos resultados obtidos foram transformados em kg ha-1.

Por ocasião da colheita, foi colhido, no terço médio das plantas, o algodão em caroço de 30 capulhos, um por planta, e acondicionados em sacos de papel, de acordo com a metodologia proposta por Sabino et al. (1975); posteriormente, e em condições de laboratório, foram realizadas as determinações de: peso médio de um capulho (g), percentagem de fibras e peso de 100 sementes (g).

Em ambos os anos agrícolas, os resultados obtidos foram submetidos à análise de variância, de acordo com Farias (1984) e Banzatto \& Kronka (1992). Para as variáveis em que o teste $\mathrm{F}$ indicou haver diferenças significativas entre os tratamentos, foi feita a análise de regressão polinomial.

\section{RESULTADOS E DISCUSSÃO}

No ano de 1993/94, o número de maçãs por planta, quando da colheita, não foi influenciado significativamente pelos fatores CM, TDZ e ETH nem também, pela interação entre eles (Tabela 2); entretanto, quando do desdobramento dos graus de liberdade do fator $\mathrm{CM}$ em componentes linear, quadrático e cúbico, verificou-se efeito significativo para o componente linear, e com o aumento da dosagem de $\mathrm{CM}$, o número de maçãs por planta reduziu linearmente, fato este que se verificou no também ano de 1994/95 (Tabela 3). O menor número de maçãs no final do ciclo, em função da aplicação de $\mathrm{CM}$, é um indicativo de que o produto aumentou a precocidade das plantas. Quando se considera o ataque de insetos-praga de ocorrência tardia, como lagarta-rosada e bicudo-do-algodoeiro, esse efeito do CM é muito importante também, pois quando os insetos-praga atingem elevadas populações a produção já está definida; outra vantagem é que a colheita pode ser realizada de uma única vez, e seus resultados, de acordo com Wallace et al. (1993), podem ser explicados como sendo devidos à maior retenção de frutos nas primeiras posições dos ramos frutíferos, o que se pode considerar como mais uma vantagem do produto, pois os frutos das primeiras posições são os que contribuem para a maior parte da produção (Jenkins et al., 1990).

A percentagem de desfolha na colheita, avaliada 14 dias após a aplicação do TDZ, foi significativa-

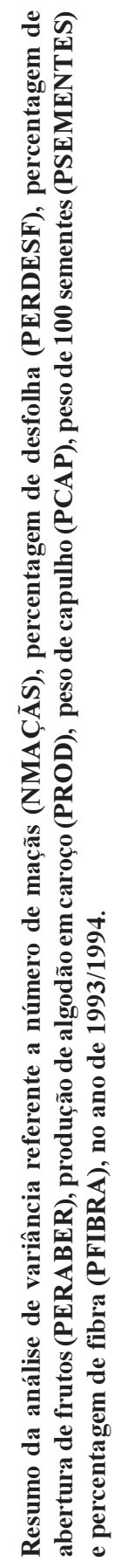

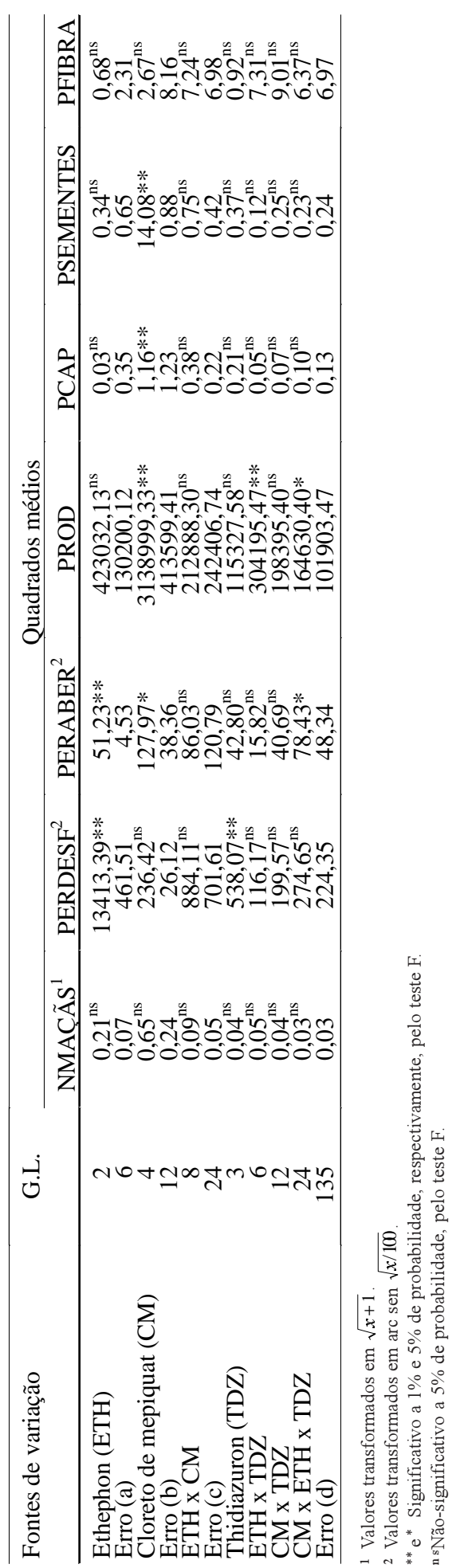


mente influenciada pelos fatores TDZ e ETH $(\mathrm{P}<0,01)$ no ano de 1993/94, e pela interação CM x TDZ $(\mathrm{P}<0,05)$ no ano de 1994/95 (Tabelas 2 e 4).

Em 1993/94, os efeitos do TDZ e do ETH foram de natureza linear, verificando-se aumento da percentagem de desfolha com o aumento da dosagem dos produtos (Tabela 3 ). No ano de 1994/95, o efeito do TDZ foi dependente da dosagem de CM, sendo significativo apenas quando foram utilizados $75,0 \mathrm{~g} \mathrm{ha}^{-1}$ de $\mathrm{CM}$, em que o efeito do TDZ sobre a percentagem de desfolha foi linear (Tabela 3). Trabalhos desenvolvidos por Paulo et al. (1989) e Malik et al. (1991), em relação ao efeito do TDZ, obtiveram resultados semelhantes.

Embora o objetivo principal da utilização do ETH na cultura do algodoeiro seja a uniformização e a aceleração da maturação dos frutos, a sua aplicação contribui para a abscisão de folhas (Warner \& Leopold, 1969), em virtude de se tratar de um produto que libera etileno.

A percentagem de abertura de capulhos foi significativamente influenciada pela interação CM x TDZ x ETH, no ano de 1993/94 (Tabela 2). Ao se analisar o efeito das doses de TDZ em função das dosagens de CM e de ETH, verifica-se, pela Fig. 1, que na ausência de CM e com $1.440 \mathrm{~g} \mathrm{ha}^{-1} \mathrm{de}$ ETH, o efeito do TDZ sobre a percentagem de abertura de capulhos foi de natureza quadrática, com ponto de máximo na dosagem de $31,72 \mathrm{~g} \mathrm{ha}^{-1}$ de
TDZ. Com $100 \mathrm{~g} \mathrm{ha}^{-1}$ de CM e $960 \mathrm{~g} \mathrm{ha}^{-1}$ de ETH, o efeito do TDZ sobre a percentagem de abertura de capulhos foi linear (Fig. 1). Malik et al. (1991), estudando apenas o efeito do TDZ, obtiveram resultados semelhantes aos obtidos neste trabalho. Paulo et al. (1989) não obtiveram efeito do TDZ, mas apenas para o ETH, na dosagem de $1.440 \mathrm{~g} \mathrm{ha}^{-1}$ ou quando se misturou TDZ com ETH. No ano de 1994/95, a percentagem de abertura de capulhos aumentou linearmente com a dosagem de CM (Fig. 2).

Com o aumento da dosagem de CM, o peso de 100 sementes aumentou linearmente no ano de 1993/94 e, de forma quadrática, no ano de 1994/95, comportamento semelhante ao verificado no tocante ao peso médio de um capulho (Tabela 3). O aumento do peso de 100 sementes com o aumento da dosagem de CM pode ser explicado em função das modificações que o produto provoca na arquitetura das plantas, conferindo-lhes maior eficiência fotossintética (Hodges et al., 1991); resultados semelhantes foram obtidos por Cia et al. (1984), Carvalho et al. (1994) e McCarty \& Hedin (1994). A ausência de efeito significativo do TDZ, do ETH e da interação, sobre os quais na literatura consultada não se encontram relatos, pode ser explicada como sendo devida ao momento em que os produtos foram aplicados, ou seja, já no final do ciclo da cultura, quando provavelmente o peso das sementes estava pouco sujeito a variações de sua matéria seca.

TABELA3. Equações de regressão para percentagem de desfolha e número de maçãs por planta, no ano de $1994 / 95$.

\begin{tabular}{|c|c|c|c|}
\hline Variável & Produto $^{1}$ & Equação & $\mathrm{R}^{2}$ \\
\hline Número de maçãs por planta $(\sqrt{x+1})$ & $\mathrm{CM}$ & $\begin{array}{l}\text { Y } 1993 / 94=1,69-0,002 X \\
\text { Y } 1994 / 95=1,42-0,0016 X\end{array}$ & $\begin{array}{l}0,70 \\
0,60 \\
\end{array}$ \\
\hline Percentagem de desfolha $(\operatorname{arcsen} \sqrt{x / 100})$ & $\begin{array}{l}\text { TDZ } \\
\text { ETH } \\
\text { TDZ + CM(75) } \\
\end{array}$ & $\begin{array}{l}\text { Y } 1993 / 94=51,81+0,29 X \\
\text { Y } 1993 / 94=50,59+0,018 X \\
\text { Y } 1994 / 95=51,10+0,14 X\end{array}$ & $\begin{array}{l}0,96 \\
0,99 \\
0,97 \\
\end{array}$ \\
\hline Peso de 100 sementes (g) & $\mathrm{CM}$ & $\begin{array}{l}\text { Y } 1993 / 94=9,64+0,011 X \\
\text { Y } 1994 / 95=9,83+0,018 X-0,0001 X^{2}\end{array}$ & $\begin{array}{l}0,96 \\
0,96 \\
\end{array}$ \\
\hline Peso médio de capulho (g) & $\mathrm{CM}$ & $\begin{array}{l}\text { Y } 1993 / 94=4,54+0,003 X \\
\text { Y } 1994 / 95=5,91+0,01 X-0,00006 X^{2}\end{array}$ & $\begin{array}{l}0,88 \\
0,91\end{array}$ \\
\hline
\end{tabular}

${ }^{1}$ TDZ: Thidiazuron; ETH: Ethephon; CM:Cloreto de mepiquat 


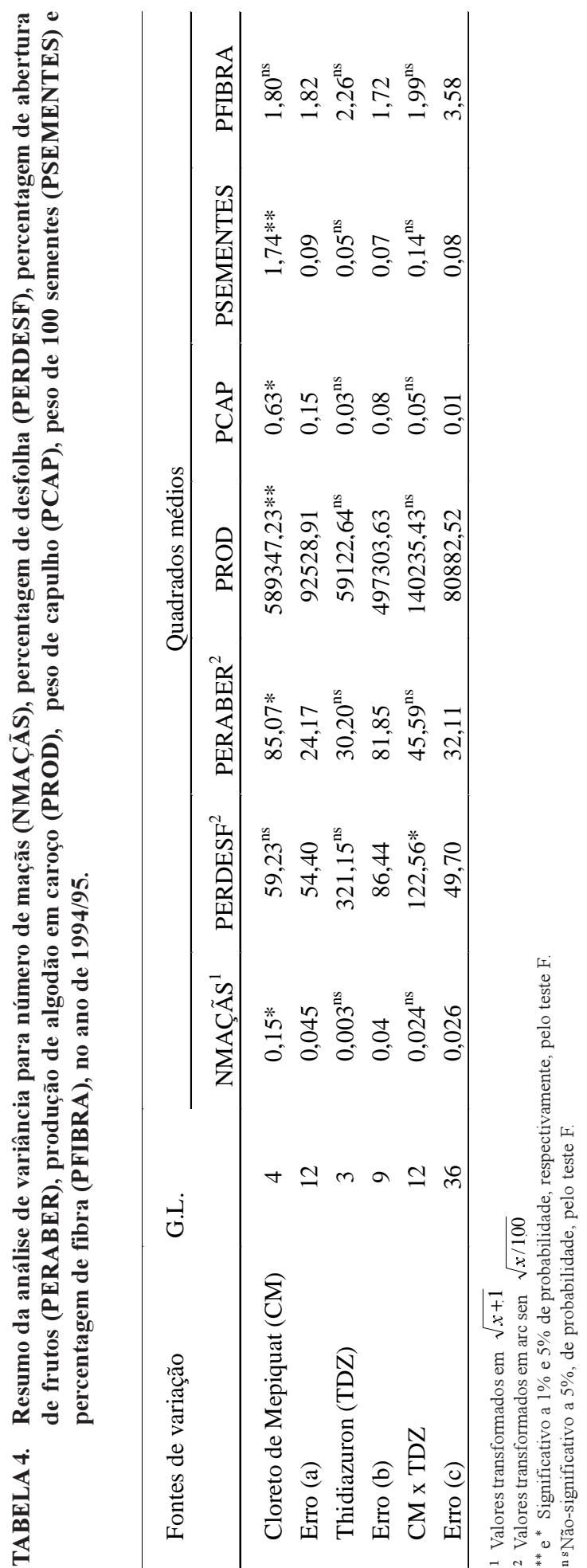

Durante o período do estudo, apenas o CM teve efeito significativo sobre o peso médio de um capulho (Tabela 3); no primeiro ano, o efeito foi linear, e no segundo, quadrático. De acordo com Stuart et al. (1984), Marani \& Ephrath (1985) e Hodges et al. (1991), plantas tratadas com CM têm a eficiência fotossintética aumentada, e também, em função do menor crescimento das plantas, maior quantidade de fotoassimilados é carreada para sua parte reprodutiva, o que as tornam mais eficientes do ponto de vista agronômico (Meredith Junior \& Wells, 1989; Reddy et al., 1990). O efeito do CM sobre o peso de 100 sementes explica o aumento do peso médio de um capulho em função da aplicação do CM.

Com relação ao efeito do TDZ sobre o peso médio de um capulho (Tabelas 2 e 4), os resultados deste trabalho são semelhantes aos de Cruz et al. (1977). Pettigrew et al. (1992) encontraram, quanto ao $\mathrm{ETH}$, resultados diferentes dos obtidos no presente trabalho, pois, de acordo com os autores, o ETH provocou redução do peso médio de um

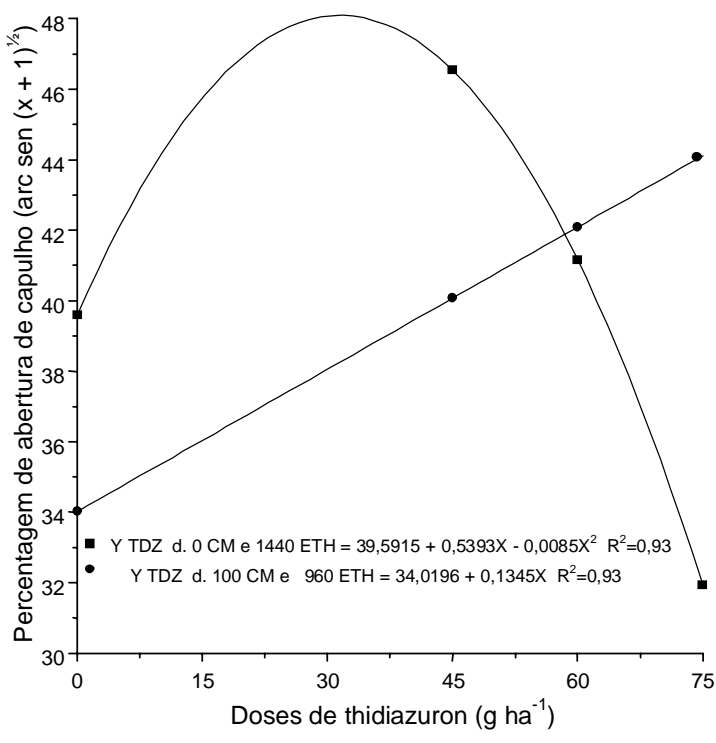

FIG. 1. Efeito das dosagens de thidiazuron na percentagem de abertura de capulho, em função das combinações de dosagens de cloreto de mepiquat e ethephon. Ponta Porã, MS, 1993/94. 
capulho; entretanto, eles fizeram aplicações aos $56 \mathrm{DAE}$, o que corresponde aproximadamente ao início do florescimento.

A interação CM x TDZ x ETH, com relação à variável produção de algodão em caroço, no ano de 1993/94, foi significativa (Tabela 2). Optou-se por se discutir o efeito do TDZ dentro das dosagens de $\mathrm{CM}$ e de ETH, em função do maior número de grau de liberdade do resíduo. Nas combinações $50 \mathrm{~g}$ de CM x $1.440 \mathrm{~g}$ de ETH e $100 \mathrm{~g}$ de CM x $0 \mathrm{~g}$ de ETH, o efeito do thidiazuron foi linear (Fig. 3), mas para as combinações de $100 \mathrm{~g}$ de CM e $1.440 \mathrm{~g}$ de ETH e $125 \mathrm{~g}$ de CM e $960 \mathrm{~g}$ de ETH, o efeito do TDZ foi quadrático, com pontos de máximo nas dosagens estimadas de 31,6 e 34,6 $\mathrm{g} \mathrm{ha}^{-1}$, respectivamente (Fig. 3). Pelos resultados obtidos, fica evidente, com relação à variável produção de algodão em caroço, que o efeito de cada um dos produtos estudados depende do produto utilizado e da dosagem. O efeito do TDZ sobre a produção de algodão em caroço foi estudado por Bariola et al. (1990) que verificaram efeito significativo apenas quando o produto foi aplicado precocemente, e provocou queda de produção; já o efeito do ETH sobre a

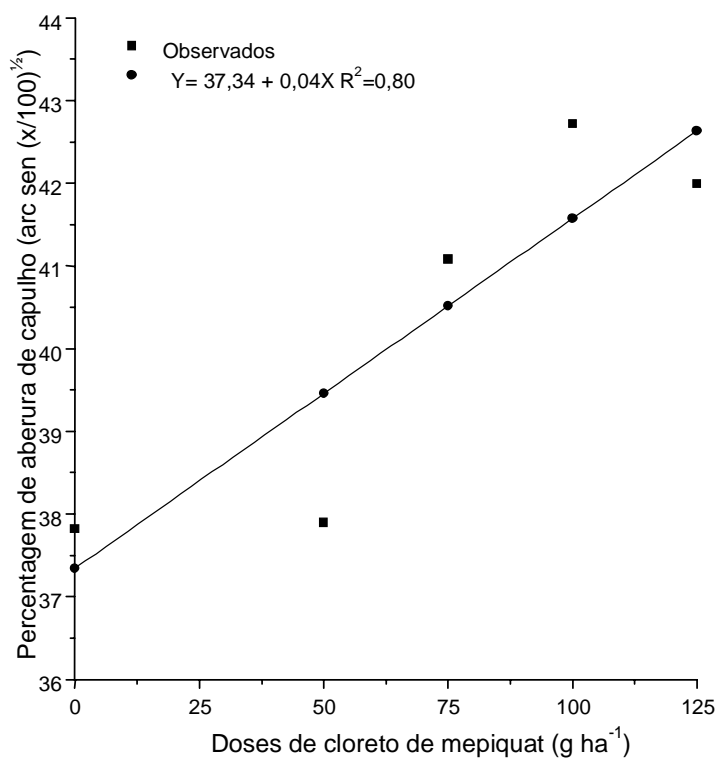

FIG. 2. Efeito das dosagens de cloreto de mepiquat na percentagem de abertura de capulho. Ponta Porã, MS, 1994/95. produção de algodão em caroço foi estudado por Jones et al. (1990), que fizeram aplicações do produto na fase final de floração, e não encontraram efeito significativo sobre a produção de algodão em caroço; entretanto, Pettigrew et al. (1992), realizando aplicações precoces aos 56 DAE, verificaram efeito depressivo do produto sobre a produção de algodão em caroço.

No ano de 1994/95, a produção de algodão em caroço correspondeu, de forma quadrática, às dosagens de $\mathrm{CM}$, com ponto de máximo na dosagem de $98,57 \mathrm{~g} \mathrm{ha}^{-1}$ (Fig. 4); este aumento pode ser explicado como tendo sido causado pela redução do número de maçãs, pelo aumento do peso de 100 sementes e pelo peso médio de um capulho (Tabela 4), pois estes são alguns dos componentes do rendimento.

O efeito do CM isolado sobre a produção de algodão em caroço é dependente de vários fatores: da cultivar, da época de semeadura, da população de plantas, da época de aplicação e das condições

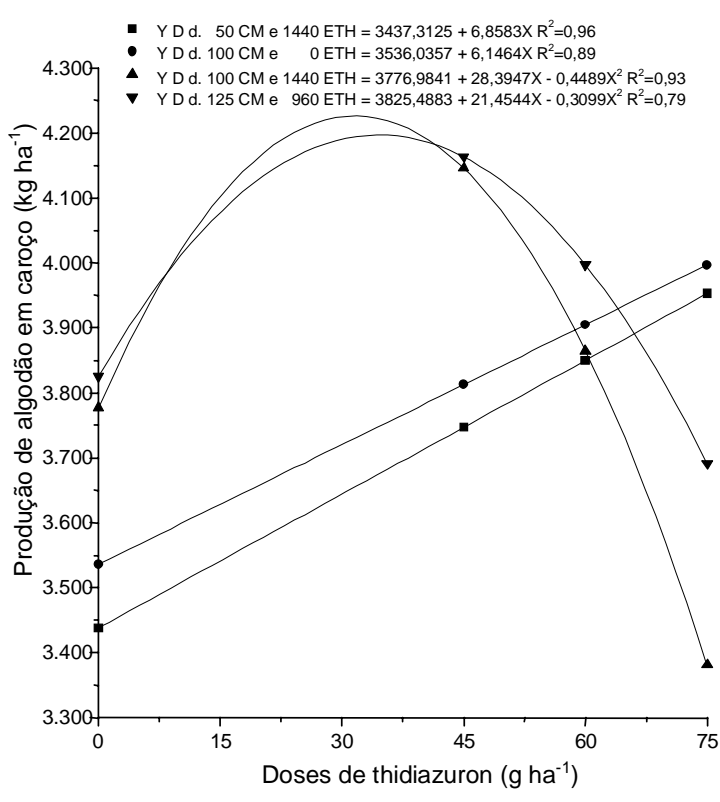

FIG. 3. Efeito das dosagens de thidiazuron na produção de algodão em caroço, em função de diferentes combinações entre cloreto de mepiquat e ethephon. Ponta Porã, MS, 1993/94. 
ambientais (York, 1983; Cathey \& Meredith Junior, 1988; Reddy et al., 1992; Wallace et al., 1993; Carvalho et al., 1994; McCarty \& Hedin, 1994). Alguns autores encontraram efeitos significativos, e outros não, o que foi explicado como tendo sido devido às condições em que os trabalhos foram desenvolvidos. O efeito do TDZ sobre a produção de algodão em caroço não foi significativo; resultados semelhantes foram obtidos por Cruz et al. (1977), Paulo et al. (1989) e Bariola et al. (1990).

A percentagem de fibras não foi influenciada significativamente pelos produtos estudados, quer de forma isolada, quer pela interação dos mesmos (Tabelas 2 e 4). Nos trabalhos desenvolvidos por Cia et al. (1984) e McCarty \& Hedin (1994), a percentagem de fibras decresceu, em decorrência da aplicação de CM. Carvalho et al. (1994) compararam o efeito do cloreto de mepiquat, do cloreto de clorocolina e do cloreto de chlormequat, e verificaram que apenas o último teve efeito significativo sobre a percentagem de fibra, provocando sua redução. Athayde (1980) constatou redução na percentagem de fibra em função da aplicação de cloreto de clorocolina.

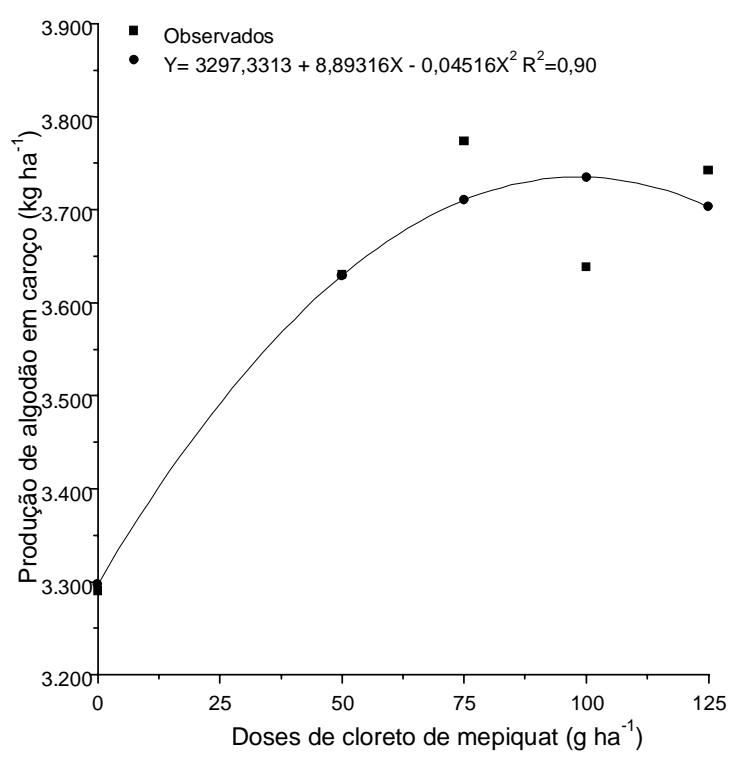

FIG. 4. Efeito das dosagens de cloreto de mepiquat na produção de algodão em caroço. Ponta Porã, MS, 1994/95.

Pesq. agropec. bras., Brasília, v.34, n.10, p.1871-1880, out. 1999

\section{CONCLUSÕES}

1. O cloreto de mepiquat proporciona redução do número de frutos verdes e aumento do peso de 100 sementes e do peso médio de um capulho.

2. A percentagem de desfolha aumenta com as dosagens de thidiazuron e ethephon.

3. A percentagem de abertura de capulhos depende das dosagens de cloreto de mepiquat, thidiazuron e ethephon.

4. Para a produção de algodão em caroço, a interação cloreto de mepiquat, thidiazuron e ethephon, é significativa.

\section{REFERÊNCIAS}

ATHAYDE, M.L.F. Efeitos de $\mathbf{N}$ e de cloreto de clorocolina $(\mathrm{CCC})$ no metabolismo nitrogenado e em algumas características do algodoeiro (Gossypium hirsutum L.). Piracicaba: USP-ESALQ, 1980. 94p. Tese de Doutorado

BANZATTO, D.A.; KRONKA, S.N. Experimentação agrícola. 2.ed. Jaboticabal: FUNEP, 1992. 247p.

BARIOLA, L.A.; CHU, C.C.; HENNEBERRY, T.J. Timing applications of plant growth regulators and last irrigation for pink bollworm (Lepidoptera: Gelechiidae) control. Journal of Economic Entomology, Lanhan, v.83, n.3, p.1074-1079, 1990

BARIOLA, L.A.; HENNEBERRY, T.J. Plant growth regulators for pink bollworm and boll weevil control. In: BELTWIDE COTTON PRODUCTION RESEARCH CONFERENCE, 1., 1986, Las Vegas. Proceedings. Memphis: National Cotton Council, 1986. p.235-238.

CARVALHO, L.H.; CHIAVEGATO, E.J.; KONDO, J.I.; SABINO, J.C.; PETTINELLI JUNIOR, A.; BORTOLETTO, N.; GALLO, P.B. Fitorreguladores de crescimento e capação na cultura do algodoeiro. Bragantia, Campinas, v.53, n.2, p.247-254, 1994.

CATHEY, G.W.; MEREDITH JUNIOR, W.R. Cotton response to planting date and mepiquat chloride. Agronomy Journal, Madison, v.80, n.3, p.463-469, 1988.

CIA, E.; CARVALHO, L.H.; KONDO, J.I.; FUZATTO, M.G.; BORTOLETTO, N.; GALLO, P.B.; CRUZ, L.S.P.; SABINO, N.P.; PETTINELLI JUNIOR, A.; 
MARTINS, A.L.M.; SILVEIRA, J.C.O. Efeito do cloreto de clorocolina e cloreto de mepiquat na cultura do algodão (Gossypium hirsutum L.). Planta Daninha, Campinas, v.2, n. 7, p.23-36,1984

CRUZ, L.S.P.; LEIDERMAN, L.; GROSSI, J.M.M. Efeito de dois novos desfolhantes para algodão (Gossypium hirsutum L.). O Biológico, Campinas, v.43, p.179-186, 1977.

FARIAS, E.H.S. Análise de experimento em faixas com subparcelas subdivididas. Piracicaba: USP-ESALQ, 1984. 81p. Dissertação de Mestrado.

HENNEBERRY, T.J.; MENG, T.; HUTCHISON, W.D.; BARIOLA, L.A.; DETIER, B.I. Effects of ethephon on boll weevil (Coleoptera: Curculionidae) population development cotton fruiting and boll opening. Journal of Economic Entomology, Lanhan, v.81, n.2, p.628-633,1988.

HODGES, H.F.; REDDY, V.R.; REDDY, K.R. Mepiquat chloride and temperature effects on photosynthesis and respiration of fruiting cotton. Crop Science, Madison, v.31, n.5, p.1302-1308,1991.

HOPKINS, A.R.; MOORE, R.F. Thidiazuron:effect of applications on boll weevil and bollworm population densities, leaf abscission and growth of the cotton plant. Journal of Economic Entomology, Lanhan, v.73, n.6, p.768-770,1980.

JENKINS, J.N.; McCARTY, J.C.; PARROTT, W.L. Effectiveness of fruiting sites in cotton: yield. Crop Science, Madison, v.30, n.2, p.368-369, 1990.

JONES, R.G.; BANER, P.J.; ROOF, M.E.; LANGSTON, M.A. Effect of reduced rates of ethephon on late-season insect oviposition and freeding sites in cotton. Journal of Entomological Science, Griffin, v. 25, n.2, p.246-252, 1990

KERBY, T.A.; JOHNSON, S.; YAMADA, H. Efficacy of cotton defoliantes. California Agriculture, Berkeley, v.38, n.9, p.24-25, 1988

MALIK, M.N.; SHABAB, U.D.; MAKHDUM, M.I. Accelerated cotton boll dehiscence with thidiazuron. Tropical Agriculture, St. Augustine, v.68, n.2, p.149-150, 1991

MARANI, A.; EPHRATH, J. Penetration of radiation into cotton crop canopies. Crop Science, Madison, v. 25, n.2, p.309-313, 1985
McCARTY, J.C.; HEDIN, P.A. Effects of 1,1 - dimethyl piperidinium chloride on the yields, agromic traits, and alelochemicals of cotton (Gossypium hirsutum L. ), a nine year study. Journal of Agricultural and Food Chemistry, Washington, DC, v.42, n.10, p.2302-2304, 1994.

MEREDITH JUNIOR, W.R.; WELLS, R. Potential for increasing cotton yield through enhanced partitioning to reproductive structures. Crop Science, Madison, v.29, n.3, p.636-639,1989.

MONTANDON, R.; SLOSSER, J.E.; CLARK, L.E. Late season termination effects on cotton fruiting, yield, and boll weevil (Coleoptera: Curculionidae) damage in Texas dryland cotton. Journal of Economic Entomology, Lanhan, v.87, n.6, p.1647-1652, 1994.

PAULO, E.M.; FUJIWARA, M.; DODO, S. Utilização do ethephon e do thidiazuron na desfolha do algodoeiro e na deiscência de seus frutos. Bragantia, Campinas, v.48, n.2, p.215-221, 1989.

PETTIGREW, W.T.; HEITHOLT, J.J.; MEREDITH JUNIOR, W.R. Early season floral bud removal and cotton growth, yield and fiber quality. Agronomy Journal, Madison, v.84, n.2, p.209-214, 1992.

REDDY, V.R.; BAKER, D.N.; HODGES, H.F. Temperature and mepiquat chloride on cotton canopy architecture. Agronomy Journal, Madison, v.82, n.2, p.190-195, 1990.

REDDY, V.R.; TRENT,A.; ACOCK, B. Mepiquat chloride and irrigation versus cotton growth and development. Agronomy Journal, Madison, v.84, n.6, p.930933,1992 .

SABINO, N.P.; LAZARINI, J.F.; GRIDI-PAPP, I.L.; FUZATTO, M.G.; GROSSI, J.M.M. Estudo de amostragens de capulhos em canteiros experimentais de algodão. Bragantia, Campinas, v.34, n.8, p.6369,1975

SANTOS, W.J. Pragas do algodoeiro. In: IAPAR Recomendações para a cultura do algodoeiro no Paraná. Londrina, 1993. p.37-63. (IAPAR. Informe da Pesquisa, 107).

SMITH, C.W.; COTHREN, J.T.; VARVIL, J.J. Yield and fiber quality of cotton following application of 2-chlorethyl phosphonic acid. Agronomy Journal, Madison, v.78, n.5, p.814-818, 1988.

SNIPES, C.E.; BASKIN, C.C. Influence of early defoliation on cotton yield, seed quality and fiber properties.

Pesq. agropec. bras., Brasília, v.34, n.10, p.1871-1880, out. 1999 
Field Crops Research, Amsterdam, v.37, n.2, p.137-143, 1994.

STUART, B.L.; ISBELL, V.R.; WENDT, C.W.; ABERNATY, J.R. Modification of cotton relations and growth with mepiquat chloride. Agronomy Journal, Madison, v.76, n.4, p.651-654,1984.

SUTTLE, J.C. Cytokinin-induced ethylene biosynthesis in nonsenescing cotton leaves. Plant Physiology, Rockville, v.78, n.2, p.272-276,1985.

SUTTLE, J.C.; HULTSTRAND, J.F. Ethylene-induced leaf abscission in cotton seedlings. Plant Physiology, Rockville, v.95, n.1, p.29-33, 1990.
THAKRAL, S.K. Effect of defoliant on upland cotton (Gossypium hirsutun L.). Journal of Agricultural Science, New Delhi, v.61, n.10, p.772-773, 1991.

WALLACE, T.P.; SNIPES, C.E.; WHITE, B.W. Effects of single -multiple applications of mepiquat chloride on Mississipi cotton. Research Reports Mississipi Agricultural Forestry Experiment Station, Mississipi, v.18, n.5, p.5, 1993.

WARNER, H.L.; LEOPOLD, A.C. Ethylene evolution from 2-chloroethyl phosponic acid. Plant Physiology, Rockville, v.44, n.1, p.156-158, 1969.

YORK, A.C. Cotton cultivar response to mepiquat chloride. Agronomy Journal, Madison, v.75, n.4, p.663-667, 1983. 